\title{
A Class of Algebraically Special Perfect Fluid Space-Times`
}

\author{
J. WAINWRIGHT \\ Department of Applied Mathematics, University of Waterloo
}

Received December 18, 1969

\begin{abstract}
Solutions of the Einstein field equations are considered subject to the assumptions that (1) the source of the gravitational field is a perfect fluid, (2) the Weyl tensor is algebraically special, (3) the corresponding repeated principal null congruence is geodesic and shearfree. If in addition, the repeated principal null congruence is nonexpanding, it follows that the twist of this congruence must be non-zero (for a physically reasonable fluid). The general line element subject to this additional restriction is derived. Furthermore, it is shown that all solutions of the Einstein field equations which satisfy (1) and exhibit local rotational symmetry, necessarily satisfy (2) and (3).
\end{abstract}

\section{§ 1. Introduction}

The Einstein field equations with a perfect fluid as source read

$$
R_{a b}-\frac{1}{2} R g_{a b}+C g_{a b}=-\left[(A+p) u_{a} u_{b}-p g_{a b}\right], \quad u_{a} u^{a}=1,
$$

where $u_{a}$ is the velocity of the fluid, $A>0$ the energy density, $p$ the scalar pressure and $C$ the cosmological constant. The metric tensor $g_{a b}$ $(a, b, c \ldots=1,2,3,4)$ has signature $(---+)$ and the conventions for the Riemann and Ricci tensors are

$$
v_{a ; b c}-v_{a ; c b}=v_{d} R_{a b c}^{d}, \quad R_{a b}=R_{a b c}^{c} .
$$

Exact solutions of (1.1) are of interest in the following connections:

(1) as cosmological models,

(2) as interior solutions to be matched to exterior vacuum solutions,

(3) as representing the propagation of gravitational radiation in matter.

Algebraically special solutions of (1.1) (i.e., solutions in which the Weyl tensor has a repeated principal null direction, cf. for example Pirani [1]) have recently been studied in each of these three contexts.

\footnotetext{
* This work was supported in part by the National Research Council of Canada.
} 
Ellis [2] and Stewart and Ellis [3] have classified the locally rotationally symmetric solutions of (1.1), which may be characterized by the fact that they admit a multiply transitive group of motions. All solutions in this class are algebraically special, and particular examples, which are nonisotropic generalizations of the Friedmann solutions, have been studied as cosmological models e.g. Kantowski and Sachs [4]. Wahlquist [5] has given an algebraically special solution of (1.1) which is of interest from the point of view of (2). Finally Szekeres [6] discussed algebraically special solutions of (1.1) from the point of view of (3), but gave no exact solutions.

In this paper we begin a systematic study of algebraically special exact solutions of (1.1). We make the additional assumption that the repeated principal null congruence $k^{a}$ is geodesic and shearfree so that

$$
k_{a ; b} k^{b}=0, \quad\left(k_{a ; b}+k_{b ; a}\right) k^{a ; b}=\left(k_{; a}^{a}\right)^{2}
$$

in terms of an affine parameter (cf. for example Pirani [1], p. 336). In vacuo, the Goldberg-Sachs theorem [7] asserts that (1.2) is necessarily satisfied. In the presence of a perfect fluid, however, it is evident from the work of Szekeres [6] that (1.2) imposes restrictions on the shear, rotation and energy-density gradient of the fluid. There are three subclasses of solutions of (1.1) satisfying the above assumptions, depending on the expansion $\frac{1}{2} k_{; a}^{a}$ and the twist $\left[\frac{1}{2}\left(k_{a ; b}-k_{b ; a}\right) k^{a ; b}\right]^{1 / 2}$ of the repeated principal null congruence, namely
A: Zero expansion, non-zero twist,
B: Non-zero expansion, zero twist,
C: Non-zero expansion, non-zero twist.

[The twist and expansion cannot both vanish unless $A+p=0$, which we exclude, cf. §3.]

Most of this paper deals with class $A$. We make use of the NewmanPenrose formalism [7] which has proved effective in deriving algebraically special solutions of the vacuum field equations (cf. for example [8] and [9]). The formalism is briefly introduced in $\S 2$. In $\S 3$ the null tetrad is adapted to the repeated principal null congruence and the velocity of the fluid, and it is shown that for the class A the parameter along $k^{a}$ can be chosen so that

$$
k_{a ; b}+k_{b ; a}=f k_{a} k_{b},
$$

for some function $f$. A coordinate system is introduced in $\S 4$, and the general line elements corresponding to $f=0$ and $f \neq 0$ are derived in $\S 5$ and $\S 6$. Finally, in $\S 7$, it is shown that the locally rotationally sym- 
metric fluid solutions form a subclass of the algebraically special fluid solutions whose repeated principal null congruence is geodesic and shearfree.

\section{§ 2. The Newman-Penrose Formalism}

Following Newman and Penrose [7] we introduce a complex null tetrad $\left(k^{a}, n^{a}, m^{a}, \bar{m}^{a}\right)$, where $k^{a}, n^{a}$ are real null vectors and $m^{a}$ is a complex null vector, with $\bar{m}^{a}$ its complex conjugate. The orthogonality properties of these vectors are

$$
\begin{aligned}
k^{a} k_{a} & =n^{a} n_{a}=m^{a} m_{a}=\bar{m}^{a} \bar{m}_{a}=0, \\
k^{a} m_{a} & =k^{a} \bar{m}_{a}=n^{a} m_{a}=n^{a} \bar{m}_{a}=0, \\
k^{a} n_{a} & =-m^{a} \bar{m}_{a}=1 .
\end{aligned}
$$

It follows that

$$
g^{a b}=k^{a} n^{b}+n^{a} k^{b}-m^{a} \bar{m}^{b}-\bar{m}^{a} m^{b} .
$$

The Newman-Penrose spin coefficients are defined by

$$
\begin{array}{lll}
\kappa=k_{a ; b} m^{a} k^{b}, & \pi=-n_{a ; b} \bar{m}^{a} k^{b}, & \varepsilon=\frac{1}{2}\left(k_{a ; b} n^{a} k^{b}-m_{a ; b} \bar{m}^{a} k^{b}\right), \\
\varrho=k_{a ; b} m^{a} \bar{m}^{b}, & \lambda=-n_{a ; b} \bar{m}^{a} \bar{m}^{b}, & \alpha=\frac{1}{2}\left(k_{a ; b} n^{a} \bar{m}^{b}-m_{a ; b} \bar{m}^{a} \bar{m}^{b}\right), \\
\sigma=k_{a ; b} m^{a} m^{b}, & \mu=-n_{a ; b} \bar{m}^{a} m^{b}, & \beta=\frac{1}{2}\left(k_{a ; b} n^{a} m^{b}-m_{a ; b} \bar{m}^{a} m^{b}\right), \\
\nu=-n_{a ; b} \bar{m}^{a} n^{b}, & \tau=k_{a ; b} m^{a} n^{b}, & \gamma=\frac{1}{2}\left(k_{a ; b} n^{a} n^{b}-m_{a ; b} \bar{m}^{a} n^{b}\right) .
\end{array}
$$

The complex tetrad components of the Weyl and Ricci tensors are denoted respectively by

$$
\begin{aligned}
\Psi_{0} & =-C_{a b c d} k^{a} m^{b} k^{c} m^{d}, \quad \Psi_{1}=-C_{a b c d} k^{a} n^{b} k^{c} m^{d}, \\
\Psi_{2} & =-\frac{1}{2} C_{a b c d}\left(k^{a} n^{b} k^{c} n^{d}-k^{a} n^{b} m^{c} \bar{m}^{d}\right), \\
\Psi_{3} & =C_{a b c d} k^{a} n^{b} n^{c} \bar{m}^{d}, \quad \Psi_{4}=-C_{a b c d} n^{a} \bar{m}^{b} n^{c} \bar{m}^{d} ; \\
\Phi_{00} & =-\frac{1}{2} R_{a b} k^{a} k^{b}, \quad \Phi_{11}=-\frac{1}{4} R_{a b}\left(k^{a} n^{b}+m^{a} \bar{m}^{b}\right), \\
\Phi_{01} & =-\frac{1}{2} R_{a b} k^{a} m^{b}, \quad \Phi_{12}=-\frac{1}{2} R_{a b} n^{a} m^{b}, \\
\Phi_{02} & =-\frac{1}{2} R_{a b} m^{a} m^{b}, \quad \Phi_{22}=-\frac{1}{2} R_{a b} n^{a} n^{b}, \quad \Lambda=(1 / 24) R .
\end{aligned}
$$

The following differential operators play an essential role

$$
\begin{aligned}
& D \phi=\phi_{; a} k^{a}, \quad \Delta \phi=\phi_{; a} n^{a}, \\
& \delta \phi=\phi_{; a} m^{a}, \quad \bar{\delta} \phi=\phi_{; a} \bar{m}^{a} .
\end{aligned}
$$


The Newman-Penrose equations consist of three sets of equations involving the quantities (2.3), (2.4), (2.5) and the operators (2.6), corresponding to

(a) the Ricci identities,

(b) the commutators of the operators (2.6) acting on scalars,

(c) the Bianchi identities.

Equations (a) and (b) in their most general form are given by Newman and Penrose [7]. All three sets of equations are contained in Pirani [1] (pp. 346-351). We will make use of (a) and (b) and some of equations (c), those corresponding to $\left(R_{a}^{b}-\frac{1}{2} R \delta_{a}^{b}\right)_{; b}=0$.

\section{§ 3. Initial Simplifications of the Spin Coefficients}

The vector $k^{a}$ of the null tetrad is chosen to be tangent to the repeated principal null congruence, and $n^{a}$ is chosen to lie in the 2-space spanned by $k^{a}$ and the velocity $u^{a}$ of the fluid. Since $u^{a} u_{a}=1$, it follows that $u^{a}$ can be expressed in the form

$$
u^{a}=2^{-1 / 2}\left[B k^{a}+(1 / B) n^{a}\right] .
$$

The directions of $k^{a}$ and $n^{a}$ are now fixed. We are also using an affine parameter on $k^{a}$. Thus the tetrad freedom is:

$$
\begin{gathered}
k^{a^{*}}=R k^{a}, \quad n^{a^{*}}=(1 / R) n^{a}, \quad D R=0, \\
m^{a^{*}}=e^{i S} m^{a}, \quad \bar{m}^{a^{*}}=e^{-i S} \bar{m}^{a},
\end{gathered}
$$

where $R, S$ are real functions.

The requirement that $k^{a}$ be a repeated principal null congruence which is geodesic (with affine parameter), shearfree and non-expanding implies

$$
\Psi_{0}=\Psi_{1}=0 ; \quad \kappa=\varepsilon+\bar{\varepsilon}=\sigma=\varrho+\varrho=0
$$

(Newman and Penrose [7]). In terms of the tetrad components of $R_{a b}$ and $u_{a}$ [cf. (3.1) and (2.5)] the field equations (1.1) read:

$$
\begin{aligned}
B^{2} \Phi_{00} & =2 \Phi_{11}=\Phi_{22} / B^{2}=\frac{1}{4}(A+p) \neq 0, \\
\Phi_{01} & =\Phi_{02}=\Phi_{12}=0, \quad \Lambda=\frac{1}{24}(A-3 p+4 C) .
\end{aligned}
$$

We now derive some consequence of (3.4) and (3.5), using the NewmanPenrose equations and the tetrad freedom, without explicitly introducing a coordinate system. Firstly we may use (3.3) to set

$$
\varrho-2 \varepsilon=0 \text {. }
$$


Then some of the Newman-Penrose equations (corresponding to the Ricci identities) reduce to

$$
\begin{aligned}
& D \varrho=\varrho^{2}+\Phi_{00} \Rightarrow D \varrho=0, \quad \Phi_{00}=-\varrho^{2} \neq 0, \\
& D \tau=(2 \tau+\bar{\pi}) \varrho, \\
& D \alpha=\frac{1}{2}(3 \pi+2 \bar{\tau}) \varrho, \\
& D \beta=\frac{1}{2}(\bar{\pi}+2 \tau) \varrho, \\
& \delta \varrho=\varrho(\bar{\alpha}+\beta+2 \tau), \\
& \delta \tau=\bar{\lambda} \varrho+\tau(\tau+\beta-\bar{\alpha}), \\
& D \lambda-\bar{\delta} \pi=\lambda \varrho+\pi(\pi-\bar{\beta}+\alpha), \\
& \Delta \varrho-\bar{\delta} \tau=-\varrho \bar{\mu}+(\bar{\beta}-\alpha-\bar{\tau}) \tau+(\gamma+\bar{\gamma}) \varrho-\Psi_{2}-2 \Lambda, \\
& \delta \alpha-\bar{\delta} \beta=\mu \varrho+\alpha \bar{\alpha}+\beta \bar{\beta}-2 \alpha \beta+2 \gamma \varrho+\frac{1}{2} \varrho(\mu-\bar{\mu})-\Psi_{2}+\Lambda+\Phi_{11} .
\end{aligned}
$$

In addition

$$
(\delta D-D \delta) Q=(\bar{\alpha}+\beta-\bar{\pi}) D Q
$$

for an arbitrary scalar function $Q$.

The twist of the congruence $k^{a}$ is proportional to $\varrho$, and thus by (3.6) is non-zero. The commutator (3.15) is applied to $\varrho$. By means of (3.6)-(3.10) this results in

$$
D(\bar{\alpha}+\beta+2 \tau)=0=4 \tau+\bar{\pi} .
$$

We may thus use (3.2) to achieve

$$
\bar{\alpha}+\beta+2 \tau=0 \Rightarrow \delta \varrho=0 .
$$

The commutator $(\delta \bar{\delta}-\bar{\delta} \delta) \varrho=0$ yields $\Delta \varrho=0$. Thus $\varrho$ is a constant, and by means of (3.2) with $R$ a constant, may be reduced to

$$
\varrho=i \Rightarrow \Phi_{00}=1, \quad B^{2}=\frac{1}{4}(A+p),
$$

by virtue of (3.5) and (3.6). The commutator (3.15) is applied to $\tau$ and, with the aid of (3.7), (3.11), (3.12), (3.16) and (3.17), reduces to $3 \varrho^{2} \bar{\lambda}=0$, so that

$$
\lambda=0 .
$$

By comparison of (3.12) and (3.13) using (3.16), one infers that

$$
\tau=\pi=0 \Rightarrow \bar{\alpha}+\beta=0=D \alpha=D \beta .
$$

At this stage, it is easily shown that $k_{a}$ satisfies an equation of the form (1.3). By means of (3.13) and the simplifications so far achieved, Eq. (3.14) 
reduces to

$$
\delta \alpha+\bar{\delta} \bar{\alpha}=4 \alpha \bar{\alpha}+i(\gamma-\bar{\gamma})+\frac{i}{2}(\mu-\bar{\mu})+i(\mu+\bar{\mu})+3 \Lambda+\Phi_{11} .
$$

The imaginary part of this equation is $\mu+\bar{\mu}=0$. The Newman-Penrose equations for $D \mu, D \gamma$ imply that $D(\gamma-\bar{\gamma}-\mu)=0$, which permits us to use (3.3) with $D S=0$ [so that $\varrho-2 \varepsilon=0$ is preserved] to achieve

$$
\mu=\gamma-\bar{\gamma} .
$$

The remaining information to be obtained from the Ricci identities is contained in the following equations

$$
\begin{aligned}
\delta \alpha+\bar{\delta} \bar{\alpha}-4 \alpha \bar{\alpha} & =2 i \mu+\frac{1}{4}(A-p+2 C), \\
D(\gamma+\bar{\gamma}) & =2 i \mu+p-C, \\
D \mu & =i(\gamma+\bar{\gamma}), \\
D \nu-\bar{\delta} \mu & =i \nu=\bar{\delta}(\gamma+\bar{\gamma}), \\
\delta \nu-2 \bar{\alpha} \nu & =\mu^{2}+B^{4}+\Delta \mu+(\gamma+\bar{\gamma}) \mu, \\
\Delta \alpha & =0=\Delta \beta,
\end{aligned}
$$

where we have substituted for $\Phi_{11}$ etc. from (3.5). In addition the nonzero tetrad components of the Weyl tensor are given by

$$
\begin{aligned}
& \Psi_{2}=i \mu-\frac{1}{12}(A-3 p+4 C)+i(\gamma+\bar{\gamma}), \\
& \Psi_{3}=D v+i v, \quad \Psi_{4}=-\bar{\delta} v+2 \alpha v .
\end{aligned}
$$

The contracted Bianchi identities yield additional information, which with the simplifications achieved so far [in particular (3.5)] assumes the form

$$
\begin{gathered}
\delta p=-2 \bar{v}, \\
(A+p) D(A+p)+2 \Delta(A-p)=0
\end{gathered}
$$

(Pirani [7], p. 351).

\section{§ 4. The Coordinate System and Field Equations}

A coordinate system is introduced, with $x^{3}$ being an affine parameter along the congruence $k^{a}$, while $x^{1}, x^{2}, x^{4}$ remain constant along $k^{a}$. Then the tetrad vectors have the form

$$
\begin{aligned}
k^{a} & =\delta_{3}^{a}, \quad n^{a}=X^{A} \delta_{A}^{a}+U \delta_{3}^{a}+V \delta_{4}^{a}, \\
m^{a} & =\xi^{A} \delta_{A}^{a}+w^{*} \delta_{3}^{a}+v^{*} \delta_{4}^{a}, \quad A=1,2,
\end{aligned}
$$

where $X^{A}, U, V$ are real and $\xi^{A}, w^{*}, v^{*}$ are complex. 
The form $k^{a}=\delta_{3}^{a}$ is preserved under the following coordinate transformation:

$$
\begin{aligned}
\zeta^{\prime} & =\zeta^{\prime}\left(\zeta, \bar{\zeta}, x^{4}\right), \quad \zeta=x^{1}+i x^{2}, \\
x^{3^{\prime}} & =x^{3}+f\left(\zeta, \bar{\zeta}, x^{4}\right), \\
x^{4^{\prime}} & =x^{4^{\prime}}\left(\zeta, \bar{\zeta}, x^{4}\right) .
\end{aligned}
$$

With the simplifications of $\S 3$, the commutators read (Newman and Penrose [7], p. 570)

$$
\begin{aligned}
(\Delta D-D \Delta) \phi & =(\gamma+\bar{\gamma}) D \phi, \quad(\delta D-D \delta) \phi=0, \\
(\delta \Delta-\Delta \delta) \phi & =-\bar{v} D \phi, \\
(\bar{\delta} \delta-\delta \bar{\delta}) \phi & =(-2 \mu D-2 i \Delta-2 \bar{\alpha} \bar{\delta}+2 \alpha \delta) \phi .
\end{aligned}
$$

By choosing $\phi\left(x^{a}\right)$ to be successively $x^{1}, x^{2}, x^{3}, x^{4}$, we obtain equations relating the components of the tetrad vectors [and hence of the metric tensor, through (2.2)], the spin coefficients and the operators (2.6). For example, with $\phi=x^{1}, x^{2}$, the first commutator yields $D X^{A}=0$. Thus the transformation (4.2) may be used to set $X^{A}=0$, provided $V \neq 0$ [we can always choose $x^{4}$ so that $V \neq 0$ ]. The function $V$ is restricted by $D V=0$, so that (4.4) may be used to achieve $V=1$. The functions $\xi^{A}$ have to satisfy

$$
\begin{aligned}
D \xi^{A} & =0=\Delta \xi^{A}, \\
\bar{\delta} \xi^{A}-\delta \bar{\xi}^{A} & =-2 \bar{\alpha} \bar{\xi}^{A}+2 \alpha \xi^{A},
\end{aligned}
$$

i.e., the $\xi^{A}$ depend only on $\zeta, \bar{\zeta}$. Thus the transformation (4.2), with $\partial \zeta^{\prime} / \partial x^{4}=0$ [so that $X^{A}=0$ is preserved] may be used to transform $\xi^{1}+i \xi^{2}=0$, i.e., $\xi^{1}=P, \xi^{2}=i P$, where $P$ is a complex function of $\zeta, \bar{\zeta}$ which by (4.6) satisfies

$$
\bar{\delta} P=2 \alpha P .
$$

For convenience we write $w^{*}=P w, v^{*}=P v$. Then the tetrad vectors (4.1) simplify to

$$
\begin{aligned}
k^{a} & =\delta_{3}^{a}, \quad n^{a}=U \delta_{3}^{a}+\delta_{4}^{a}, \\
m^{a} & =P\left(\delta_{1}^{a}+i \delta_{2}^{a}+w \delta_{3}^{a}+v \delta_{4}^{a}\right) .
\end{aligned}
$$

The restrictions on $v$ arising from the commutators are

$$
D v=0=\Delta v, \quad P \bar{\delta} v-\bar{P} \delta \bar{v}=-2 i .
$$

This implies $v=v(\zeta, \bar{\zeta})$, so that we may use (4.4) with $\partial x^{4^{\prime}} / \partial x^{4}=1$ to achieve

$$
v+\bar{v}=0 \Rightarrow P \bar{P} \partial v / \partial x^{1}=-i
$$


by (4.9). Thus $v$ is completely determined in terms of $P$ since the "constant" of integration arising in (4.10) may be eliminated using $x^{4^{\prime}}=x^{4}+g\left(x^{2}\right)$.

The restrictions on the remaining functions $w, U$ arising from the commutators are

$$
\begin{gathered}
D w=0, \quad D U=-(\gamma+\bar{\gamma}), \\
\delta U=P \Delta w-\bar{v}, \\
P \bar{\delta} w-\bar{P} \delta \bar{w}=-2(\mu+i U) .
\end{gathered}
$$

It is convenient to define a new operator $\delta^{*}$ by

$$
\delta \phi=P \delta^{*} \phi .
$$

We now combine Eqs. (4.7), (4.11) and (4.12) with Eqs. (3.19) to (3.23) and (3.26) and arrive at the following set of equations

$$
\begin{gathered}
D^{2} U=-2 i \mu-p+C, \\
D(\mu+i U)=0, \\
(\mu D+i \Delta)(2 i \mu-p)=0, \\
(A+p) D(A+p)+2 \Delta(A-p)=0, \\
2 P \bar{P} \partial^{2}(\ln P \bar{P}) / \partial \zeta \partial \bar{\zeta}=2 i \mu+\frac{1}{4}(A-p+2 C), \\
P \bar{P} \delta^{*} \bar{\delta}^{*} p-(\mu D+i \Delta) p=-2\left(\mu^{2}+B^{4}\right), \\
\delta^{*}\left(U-\frac{1}{2} p\right)=\partial w / \partial x^{4}, \\
\delta^{*}[-2 \mu+i p+D p]=0 .
\end{gathered}
$$

[We include (3.27) again for completeness.] The only spin coefficient appearing in these equations is $\mu$, which is related to $w$ and $U$ according to (4.13). We now use the remaining tetrad freedom, namely (3.3) with $S=S(\zeta, \bar{\zeta})$, to set

$$
P=\bar{P} \text {. }
$$

At this stage the repeated principal null congruence $k^{a}$ satisfies

$$
k_{a ; b}=-\left(\partial U / \partial x^{3}\right) k_{a} k_{b}-i\left(m_{a} \bar{m}_{b}-\bar{m}_{a} m_{b}\right) .
$$

To complete the simplification of the field equations and line element, it is necessary to distinguish the two cases $\partial U / \partial x^{3}=0$, and $\partial U / \partial x^{3} \neq 0$. 


$$
\begin{gathered}
\text { § 5. Case I } \\
\partial U / \partial x^{3}=0 \Leftrightarrow k_{a ; b}+k_{b ; a}=0
\end{gathered}
$$

Eqs. (4.14)-(4.18) imply

$$
\begin{gathered}
2 i \mu=-p+C, \\
p=p\left(x^{1}, x^{2}\right), \quad A=A\left(x^{1}, x^{2}\right) .
\end{gathered}
$$

We now use (4.3) to set

$$
U=\frac{1}{2}(p-C) .
$$

Eq. (4.20) reduces to $\partial w / \partial x^{4}=0$, which enables us to use (4.3) with $\partial f / \partial x^{4}=0$ [so that (5.2) is preserved] to achieve

$$
w+\bar{w}=0 \Rightarrow P^{2} \partial w / \partial x^{1}=-i(p-C),
$$

by means of (4.13). The "constant" of integration which arises in (5.3) may be eliminated by means of $x^{3^{\prime}}=x^{3}+g\left(x^{2}\right)$. A straightforward calculation using (2.2), (4.8), (4.10) and (5.3) shows that the line element has the form

$$
\begin{aligned}
d s^{2}= & -\left(1 / 2 P^{2}\right)\left[\left(d x^{1}\right)^{2}+\left(d x^{2}\right)^{2}\right]-2 v(w-U v)\left(d x^{2}\right)^{2}+2 i v d x^{2} d x^{3} \\
& +2 i(w-2 U v) d x^{2} d x^{4}+2 d x^{3} d x^{4}-2 U\left(d x^{4}\right)^{2},
\end{aligned}
$$

where $v$ is given by (4.10). The remaining field equations (4.18) and (4.19) reduce to

$$
\begin{aligned}
P^{2} \partial^{2} p / \partial \zeta \partial \bar{\zeta} & =-(1 / 32)(A-p+2 C)(A+3 p-2 C), \\
P^{2} \partial^{2}\left(\ln P^{2}\right) / \partial \zeta \partial \bar{\zeta} & =(1 / 8)(A-5 p+6 C) .
\end{aligned}
$$

Note that the density $A\left(x^{1}, x^{2}\right)$ appears as an arbitrary function in these equations. The velocity $u^{a}$ of the fluid is given by (3.1) and (3.18). The motion of the fluid is described by its expansion $\theta$, acceleration $\dot{u}^{a}$, rotation $\omega_{a b}$ and shear $\sigma_{a b}$, defined (cf. for example Stewart and Ellis [3]) by

$$
\begin{aligned}
\theta & =u_{; a}^{a}, \quad \dot{u}^{a}=u_{; b}^{a} u^{b}, \\
\omega_{a b} & =u_{[a ; b]}-\dot{u}_{[a} u_{b]}, \\
\sigma_{a b} & =u_{(a ; b)}-\dot{u}_{(a} u_{b)}-\frac{1}{3}\left(g_{a b}-u_{a} u_{b}\right) \theta .
\end{aligned}
$$

It follows that in Case I, the fluid has zero expansion. Furthermore

$$
\begin{aligned}
\dot{u}^{a} & =0 \Leftrightarrow \partial p / \partial \zeta=0, \\
\omega_{a b} & =0 \Leftrightarrow A+3 p-2 C=0, \quad \partial p / \partial \zeta=0, \\
\sigma_{a b} & =0 \Leftrightarrow \partial(A+3 p) / \partial \zeta=0 .
\end{aligned}
$$


By means (3.25) one finds that the non-zero tetrad components of the Weyl tensor are

$$
\begin{aligned}
& \Psi_{2}=-\frac{1}{12}(A+3 p-2 C), \quad \Psi_{3}=-i P \partial p / \partial \zeta, \\
& \Psi_{4}=-2 \partial\left[P^{2} \partial p / \partial \zeta\right] / \partial \zeta .
\end{aligned}
$$

Thus in Case I the fluid has non-zero rotation provided that $C_{a b c d} \neq 0$.

\section{Particular Solutions}

Case $A . \partial p / \partial \zeta=0 \Leftrightarrow p=$ constant, $\dot{u}^{a}=0$.

Eq. (5.5) implies $A-p+2 C=0$, or $A+3 p-2 C=0$, so that $A$ is also constant. The constant pressure $p$ can be equated to zero by replacing $A, C$ by $A+p$ and $C+p$ respectively. Thus, in effect, we have dust solutions with cosmological term. Eqs. (4.10) and (5.3) imply

$$
\partial(w+C v) / \partial x^{1}=0,
$$

so that we can use the coordinate freedom remaining in (4.3) to transform $w+C v=0 \Rightarrow w-2 U v=0$. After a further coordinate transformation $x^{4^{\prime}}=x^{4}+x^{3} / C$, the line element reads

$$
\begin{aligned}
d s^{2}= & -\left(1 / 2 P^{2}\right)\left[\left(d x^{1}\right)^{2}+\left(d x^{2}\right)^{2}\right]+\left(v^{2} / C\right)\left(d x^{2}\right)^{2}+2 i v d x^{2} d x^{3} \\
& -(1 / C)\left(d x^{3}\right)^{2}+C\left(d x^{4}\right)^{2} .
\end{aligned}
$$

Case $A_{1} . A+2 C=0 \Rightarrow C<0$.

Write $C=-a^{2}$. Eq. (5.6) reads

$$
2 P^{2} \partial^{2}\left(\ln P^{2}\right) / \partial \zeta \partial \bar{\zeta}=-a^{2} .
$$

Under $\zeta^{\prime}=\zeta^{\prime}(\zeta)$ this has the unique solution

$$
P^{2}=\sinh ^{2} a x^{1} \Rightarrow v=(i / a) \cosh a x^{1} / \sinh a x^{1} .
$$

A coordinate transformation $x^{1^{\prime}}=x^{1^{\prime}}\left(x^{1}\right)$ with $d x^{1^{\prime}} / d x^{1}=\sinh a x^{1}$ simplifies the line element to

$$
\begin{aligned}
d s^{2}= & -\frac{1}{2}\left(d x^{1}\right)^{2}+\left(1+\frac{1}{2} \sinh ^{2} a x^{1}\right)\left(d x^{2}\right)^{2}-(2 / a) \cosh a x^{1} d x^{2} d x^{3} \\
& +\left(1 / a^{2}\right)\left(d x^{3}\right)^{2}-a^{2}\left(d x^{4}\right)^{2} .
\end{aligned}
$$

This solution is type $[2,2]$, and admits a 5-parameter group of motions multiply transitive on spacetime, and hence is the Gödel universe (Ellis [2], p. 1191).

Case $A_{2} \cdot A-2 C=0 \Rightarrow C>0$.

Write $2 C=a^{2}$. A similar procedure leads to the unique line element

$$
\begin{aligned}
d s^{2}= & -\frac{1}{2}\left[\left(d x^{1}\right)^{2}+\left(d x^{2}\right)^{2}\right]-(2 / a) \cos a x^{1} d x^{2} d x^{3}-\left(2 / a^{2}\right)\left(d x^{3}\right)^{2} \\
& +\left(a^{2} / 2\right)\left(d x^{4}\right)^{2} .
\end{aligned}
$$


This solution is conformally flat and admits a 7-parameter group of motions multiply transitive on spacetime and hence is the Einstein static universe (Ellis [2], p. 1191).

Case B. $\partial^{2} p / \partial \zeta \partial \bar{\zeta}=0, \partial p / \partial \zeta \neq 0$.

Eq. (5.5) implies $A-p+2 C=0$ or $A+3 p-2 C=0$. We consider only the first possibility and set

$$
C=0 \Rightarrow p=A,
$$

which is of the form of an equation of state

$$
p=(X-1) A
$$

corresponding to the maximum possible value of $X$, namely $X=2$ (cf. for example Ellis [2]).

A particular solution is

$$
\begin{aligned}
& p=x^{1}, \quad P^{2}=(2 / 3)\left(x^{1}\right)^{3}, \quad v=3 i / 4\left(x^{1}\right)^{2}, \\
& w=3 i / 2 x^{1}, \quad U=\frac{1}{2} x^{1} .
\end{aligned}
$$

After a coordinate transformation $x^{1}=3 /\left(x^{1}\right)^{2}, x^{2}=12 x^{2^{\prime}}$, the line element reads

$$
\begin{aligned}
d s^{2}= & -\left(d x^{1}\right)^{2}+5\left(x^{1}\right)^{6}\left(d x^{2}\right)^{2}-2\left(x^{1}\right)^{4} d x^{2} d x^{3}-6\left(x^{1}\right)^{2} d x^{2} d x^{4} \\
& +2 d x^{3} d x^{4}-\left[3 /\left(x^{1}\right)^{2}\right]\left(d x^{4}\right)^{2},
\end{aligned}
$$

with

$$
p=A=3 /\left(x^{1}\right)^{2} .
$$

This solution is of type [211] and admits a 3-parameter group of motions simply transitive on $x^{1}=$ constant. The acceleration, shear and rotation of the fluid are non-zero.

\section{§ 6. Case II}

$$
\partial U / \partial x^{3} \neq 0 \Leftrightarrow k_{a ; b}+k_{b ; a} \neq 0 .
$$

By virtue of (4.15), the coordinate freedom (4.3) may be used to transform

$$
\mu+i U=0 \text {. }
$$

Then (4.16) reduces to

$$
\partial\left(U-\frac{1}{2} p\right) / \partial x^{4}=0
$$

and (4.20) yields

$$
\partial w / \partial x^{4}=2 \partial\left(U-\frac{1}{2} p\right) / \partial \bar{\zeta}+w \partial\left(U-\frac{1}{2} p\right) / \partial x^{3} .
$$


By virtue of (4.11), differentiation with respect to $x^{3}$ and then $x^{4}$ yields

$$
0=\left(\partial w / \partial x^{4}\right)\left[\partial^{2}\left(U-\frac{1}{2} p\right) / \partial x^{3} \partial x^{3}\right] .
$$

At this stage a further subdivision becomes necessary.

Case II $a . \partial^{2}\left(U-\frac{1}{2} p\right) / \partial x^{3} \partial x^{3}=0$.

Eqs. (6.2) and (6.4) imply

$$
U-\frac{1}{2} p=b x^{3}+r(\zeta, \bar{\zeta}), \quad b=\text { constant } .
$$

With (6.1), Eq. (4.14) yields

$$
\partial^{2} p / \partial x^{3} \partial x^{3}=-4\left(p+b x^{3}+r-\frac{1}{2} C\right),
$$

which has as general solution

$$
p=\frac{1}{2}\left[R\left(\zeta, \bar{\zeta}, x^{4}\right) e^{2 i x^{3}}+\bar{R}\left(\zeta, \bar{\zeta}, x^{4}\right) e^{-2 i x^{3}}\right]-\left(b x^{3}+r\right)+\frac{1}{2} C .
$$

In terms of the notation

$$
K(\zeta, \bar{\zeta})=2 P^{2} \partial^{2}\left(\ln P^{2}\right) / \partial \zeta \partial \bar{\zeta}
$$

Eq. (4.18) can be written, with the aid of (6.1) and (6.5), as

$$
K=\frac{1}{4}(A+3 p+2 C)+2\left(b x^{3}+r\right) .
$$

By means of (6.5) and (6.8), the density $A$ can be eliminated from equation (4.17). With the aid of the expression (6.6) for $p$, the $x^{3}$-dependence in the resulting equation entails

$$
\begin{aligned}
b & =0 \Rightarrow R \neq 0, \\
\partial(\ln R) / \partial x^{4} & =2 i\left(r-K+\frac{1}{2} C\right) .
\end{aligned}
$$

At this stage, Eq. (4.21) reduces to

$$
2 \partial \ln R / \partial \bar{\zeta}+v \partial \ln R / \partial x^{4}+2 i w=0 .
$$

On differentiation with respect to $x^{4}$, this yields, with the aid of (6.3), (6.5), (6.9), (6.10) and (6.8):

$$
\partial(K-2 r) / \partial \zeta=0 \Rightarrow \partial(A+3 p) / \partial \zeta=0 .
$$

We thus write

$$
A+3 p-2 C=4 m, \quad m=\text { constant } .
$$

Eqs. (6.10) and (6.3) now read

$$
\partial(\ln R) / \partial x^{4}=-i(K+m), \quad \partial w / \partial x^{4}=\partial K / \partial \bar{\zeta} .
$$

The $x^{4}$-dependence in $p$ and $w$ can now be eliminated by a transformation

$$
x^{3^{\prime}}=x^{3}-\frac{1}{2} x^{4}(K+m)+f(\zeta, \bar{\zeta}) .
$$


In the new coordinate system Eqs. (6.13), (6.1), (6.5) and (6.11) read respectively (with the aid of (6.8), (6.9) and (6.12))

$$
\begin{gathered}
\partial w / \partial x^{4}=0=\partial R / \partial x^{4}, \quad \mu+i U=-\frac{i}{2}(K+m), \\
U=\frac{1}{2}(p-C)-m, \\
\partial(\ln R) / \partial \bar{\zeta}+i w=0 .
\end{gathered}
$$

In addition the arbitrary function $f$ in (6.14) can be chosen so that

$$
w+\bar{w}=0 \Rightarrow 2 P^{2} \partial w / \partial x^{1}=i(K+m)
$$

by (4.13) and (6.15). As in $\S 5$, the line element reduces to

$$
\begin{aligned}
d s^{2}= & -\left(1 / 2 P^{2}\right)\left[\left(d x^{1}\right)^{2}+\left(d x^{2}\right)^{2}\right]-2 v(w-U v)\left(d x^{2}\right)^{2}+2 i v d x^{2} d x^{3} \\
& +2 i(w-2 U v) d x^{2} d x^{4}+2 d x^{3} d x^{4}-2 U\left(d x^{4}\right)^{2},
\end{aligned}
$$

where $v$ is given by (4.10). The pressure $p$ is given by (6.6) which reads

$$
p=\frac{1}{2}\left[R(\zeta, \bar{\zeta}) e^{2 i x^{3}}+\bar{R}(\zeta, \bar{\zeta}) e^{-2 i x^{3}}\right]+C+\frac{1}{2}(m-K) .
$$

The function $R$ is determined by (6.17) up to an arbitrary function $f(\zeta)$. The remaining field equation (4.19) reduces to

$$
2 P^{2} \partial^{2} K / \partial \zeta \partial \bar{\zeta}=m(K+m),
$$

where $K$ is defined by (6.7).

The non-zero tetrad components of the Weyl tensor are

$$
\begin{aligned}
& \Psi_{2}=R e^{2 i x^{3}}-\frac{1}{3} m \neq 0, \\
& \Psi_{3}=-\frac{i}{2} P(3 H-\partial K / \partial \zeta), \\
& \Psi_{4}=\partial\left[P^{2}(H-\partial K / \partial \zeta)\right] / \partial \zeta+i w P^{2} H,
\end{aligned}
$$

where

$$
H=e^{2 i x^{3}}(\partial R / \partial \zeta-i w R) .
$$

Thus all solutions in this class are of type [211] or [22].

Finally Eqs. (6.17), (6.12) and (3.18) imply $U=-B^{2}$, so that the velocity of the fluid as given by (3.1) reduces to

$$
u^{a}=\left(1 / 2^{1 / 2} B\right) \delta_{4}^{a} .
$$

This shows that $u^{a}$ is collinear with a Killing vector so that the fluid has zero expansion and shear. On the other hand a straightforward calculation shows that for all solutions in this class, the fluid has non-zero acceleration and rotation. In particular there are no dust solutions in this class. In the limit $R \rightarrow 0$, we obtain the shearfree subclass of Case I. 


\section{Particular Solution}

$$
\partial^{2} K / \partial \zeta \partial \bar{\zeta}=0, \quad m>0 \Rightarrow K+m=0 .
$$

By virtue of (6.18), we can use $x^{3^{\prime}}=x^{3}+f\left(x^{2}\right)$ to obtain $w=0$. Then by means of (6.17) the expression (6.20) for $p$ reduces to

$$
p=\frac{1}{2}\left[R(\zeta) e^{2 i x^{3}}+\bar{R}(\bar{\zeta}) e^{-2 i x^{3}}\right]+C+m .
$$

Since $K<0$, we write $K=-a^{2}$. Then $P, v$ are the same as in case $\mathrm{A}_{1}$ of $\S 5$, and the coordinate transformation used there leads to

$$
\begin{aligned}
d s^{2}= & -\frac{1}{2}\left(d x^{1}\right)^{2}+\frac{1}{2}\left[1-\left(1+4 U / a^{2}\right) \cosh ^{2} a x^{1}\right]\left(d x^{2}\right)^{2} \\
& -(2 / a) \cosh a x^{1} d x^{2} d x^{3}+(4 U / a) \cosh a x^{1} d x^{2} d x^{4} \\
& +2 d x^{3} d x^{4}-2 U\left(d x^{4}\right)^{2}
\end{aligned}
$$

where $U$ is given by $(6.16)$.

The special case $R=$ constant in (6.23) is of interest. The pressure $p$ (and hence the energy density $A$ ) remains finite for all values of the coordinates $-\infty<x^{1}, x^{2}, x^{3}, x^{4}<\infty$. If $C<0$ and $C+m>0$ and $R$ is sufficiently small, the inequalities $A-p>0, p>0$ are satisfied everywhere. In the limit $R \rightarrow 0$, we obtain the Gödel solution.

This solution admits a 4-parameter group of isometries multiply transitive in $x^{3}=$ constant. It is thus one of the locally rotationally symmetric solutions found by Stewart and Ellis [3], but in a simpler coordinate system, since the coordinate transformation that relates the above line element to the form given in [3] (case Ib, with $\varepsilon E=0=\tau$ ) introduces a coordinate singularity.

Case II $b . \partial^{2}\left(U-\frac{1}{2} p\right) / \partial x^{3} \partial x^{3} \neq 0 \Rightarrow \partial w / \partial x^{4}=0$.

Eq. (4.13) with (6.1) implies $\partial w / \partial \zeta-\partial \bar{w} / \partial \bar{\zeta}=0$, so that we may use (4.3) with $\partial f / \partial x^{4}=0$ (so that (6.1) is preserved) to achieve

$$
w=0 .
$$

Then by virtue of (6.3), we may write

$$
U-\frac{1}{2} p=b\left(x^{3}\right), \text { with } \quad b^{\prime \prime} \neq 0 .
$$

By means of (6.7), (4.18), (6.1) and (6.25):

$$
\begin{aligned}
K(\zeta, \bar{\zeta}) & =\frac{1}{4}(A+3 p)+2 b+\frac{1}{2} C, \\
U+B^{2} & =K-b-\frac{1}{2} C \neq 0 .
\end{aligned}
$$

In addition, by means of (6.1) and (6.25), (4.21) yields

$$
\partial\left(\delta^{*} p\right) / \partial x^{3}=-2 i \delta^{*} p .
$$


On differentiation of (4.19) with respect to $x^{3}$, this equation gives rise to

$$
-\partial p / \partial x^{4}=\partial\left(U^{2}-B^{4}\right) / \partial x^{3}, \quad \partial^{2} p / \partial x^{3} \partial x^{4}=4\left(U^{2}-B^{4}\right) .
$$

These combine to give $\partial^{2}\left(U^{2}-B^{4}\right) / \partial x^{3} \partial x^{3}=-4\left(U^{2}-B^{4}\right)$ which has the general solution

$$
U^{2}-B^{4}=R\left(\zeta, \bar{\zeta}, x^{4}\right) e^{2 i x^{3}}+\bar{R}\left(\zeta, \bar{\zeta}, x^{4}\right) e^{-2 i x^{3}} .
$$

We now show that $R \neq 0$, i.e., $U^{2}-B^{4} \neq 0$, gives rise to a contradiction. Differentiation of (6.28) with respect to $x^{4}$ yields, by virtue of (6.29) and (6.30):

$$
2 \partial \ln R / \partial \bar{\zeta}+v \partial \ln R / \partial x^{4}=0 .
$$

By means of (6.25) to (6.27) and (6.30), the first of Eqs. (6.29) reads

$$
\begin{aligned}
-\left[\left(\partial R / \partial x^{4}\right) e^{2 i x^{3}}+\partial \bar{R} / \partial x^{4} e^{-2 i x^{3}}\right] \\
=2 i\left(K-b-\frac{1}{2} C\right)\left[R e^{2 i x^{3}}-\bar{R} e^{-2 i x^{3}}\right] .
\end{aligned}
$$

This equation is multiplied by $e^{2 i x^{3}}$ and differentiated twice with respect to $x^{3}$ to yield an equation of the form

$$
\partial \ln R / \partial x^{4}=-2 i K(\zeta, \bar{\zeta})+H,
$$

where $H$ is a complex constant. By virtue of (6.31) this entails $K=$ constant, so that

$$
R=R^{\circ}(\zeta, \bar{\zeta}) e^{r x^{4}}, \quad r=-2 i K+\mathrm{H},
$$

where by $(6.31) R^{\circ}$ satisfies

$$
2 \partial \ln R^{\circ} / \partial \bar{\zeta}+v r=0 .
$$

Substitute (6.33) in (6.32) and differentiate with respect to $x^{4}$. This yields $r=\bar{r}$, and the $x^{4}$-dependence in (6.32) cancels out. The resulting equation, when differentiated with respect to $\zeta$, results in $\partial \ln \left(R^{\circ} / \bar{R}^{\circ}\right) / \partial \zeta=0$. However, (6.34), with (4.9), implies

$$
2 P^{2} \partial^{2} \ln \left(R / R^{\circ}\right) / \partial \zeta \partial \bar{\zeta}=i r .
$$

Thus $r=0, \Rightarrow \partial R / \partial x^{4}=0 \Rightarrow R=0$ by (6.32), and the assumption $U^{2}-B^{4} \neq 0$ leads to a contradiction.

By (6.27), $U+B^{2} \neq 0$, so that we must have

$$
U-B^{2}=0 \Rightarrow A-p=4 b .
$$

Eqs. (6.29) and (6.26) entail

$$
\partial p / \partial x^{4}=0, \quad \partial K / \partial \zeta=\partial p / \partial \zeta \Rightarrow \partial^{2} p / \partial x^{3} \partial \zeta=0 \Rightarrow \partial p / \partial \zeta=0,
$$


the last assertion being a consequence of (6.28). We thus have

$$
p=p\left(x^{3}\right), \quad A=A\left(x^{3}\right), \quad K=\text { constant },
$$

and by means of (6.26) and (6.35)

$$
3 A+p=4 K-2 C \text {. }
$$

The $x^{3}$-dependence is determined by Eq. (4.14) which now reads

$$
d^{2} U / d x^{3} d x^{3}=-8 U+2 K .
$$

Under a coordinate transformation $x^{3^{\prime}}=x^{3}+d, d=$ constant, this has the general solution

$$
U\left(x^{3}\right)=\frac{1}{4} K+\frac{1}{6} p_{0} \cos 2^{3 / 2} x^{3},
$$

where $p_{0}$ is an arbitrary constant, and $K$ must be positive to ensure $B^{2}=U>0$. By means of (6.25), (6.35) and (6.37) the pressure is given by

$$
p=C-\frac{1}{2} K+p_{0} \cos 2^{3 / 2} x^{3} .
$$

We write $K=a^{2}$. Then $P, v$ are the same as in case $A_{2}$ of $\S 5$, and the coordinate transformation used there leads to

$$
\begin{aligned}
d s^{2}= & -\frac{1}{2}\left(d x^{1}\right)^{2}-\frac{1}{2}\left[1-\left(1-4 U / a^{2}\right) \cos ^{2} a x^{1}\right]\left(d x^{2}\right)^{2} \\
& -(2 / a) \cos a x^{1} d x^{2} d x^{3}+(4 U / a) \cos a x^{1} d x^{2} d x^{4} \\
& +2 d x^{3} d x^{4}-2 U\left(d x^{4}\right)^{2},
\end{aligned}
$$

where, by (6.35), $U=\frac{1}{4}(A+p)$, and the density and pressure are given by (6.37) and (6.38) with $K=a^{2}$. The velocity of the fluid, as given by (3.1), reduces to

$$
u^{a}=\left(1 / 2^{1 / 2} B\right)\left(2 U \delta_{3}^{a}+\delta_{4}^{a}\right), \quad u_{a}=\left(1 / 2^{1 / 2} B\right) \delta_{a}^{3} .
$$

The rotation and acceleration of the fluid are zero, but the expansion and shear scalar are given by

$$
\theta=\left(p_{0} / 3 B\right) \cos 2^{3 / 2} x^{3}, \quad \sigma_{a b} \sigma^{a b}=2 \theta^{3} / 3 .
$$

The Weyl tensor is type $[2,2]$, the only non-zero tetrad component being

$$
\Psi_{2}=\left(p_{0} / 9\right)\left[4 \cos 2^{3 / 2} x^{3}+3 i 2^{1 / 2} \sin 2^{3 / 2} x^{3}\right] .
$$

In addition the pressure $p$ (and hence the density $A$ ) remains finite for all values of the coordinates $-\infty<x^{1}, x^{2}, x^{3}, x^{4}<\infty$. If the inequalities $0<C<a^{2}<2 C$ are satisfied, and $p_{0}$ is sufficiently small, then the conditions $p>0, A-p>0$ will be satisfied everywhere. In the limit $p_{0} \rightarrow 0$ we obtain the Einstein static universe. 
This solution is one of the locally rotationally symmetric solutions found by Stewart and Ellis [3] (case III a, $\alpha \neq 0$, with $E=\tau=0$ ), but in a simpler coordinate system, since the coordinate transformation that relates the above line element to the form given in [3] introduces a singularity.

\section{§ 7. Locally Rotationally Symmetric Spacetimes with Perfect Fluid}

We finally prove that the locally rotationally symmetric dust and perfect fluid spacetimes of Ellis [2] and Stewart and Ellis [3] belong to the general class under consideration.

Theorem 7.1. For all locally rotationally symmetric spacetimes with a perfect fluid (or dust) which are not conformally flat, the Weyl tensor is type $[2,2]$ and the two repeated principal null congruences are geodesic and shearfree.

Proof. The general line element for locally rotationally symmetric spacetimes with perfect fluid is (Stewart and Ellis [3], p. 1074):

$$
\begin{aligned}
d s^{2} & =\left(d x^{0}\right)^{2} / F^{2}-X^{2}\left(d x^{1}\right)^{2}-Y^{2}\left[\left(d x^{2}\right)^{2}+t^{2}\left(d x^{3}\right)^{2}\right] \\
& -\left(y / F^{2}\right)\left(2 d x^{0}-y d x^{3}\right) d x^{3}+X^{2} h\left(2 d x^{1}-h d x^{3}\right) d x^{3},
\end{aligned}
$$

where $F, X, Y$ are functions of $x^{0}, x^{1}$ and $t, y, h$ are functions of $x^{2}$. The coordinates are now denoted $x^{0}, x^{1}, x^{2}, x^{3}$ in accordance with the notation of [3], but the signature is changed to $(---+)$. There are three distinct cases in which the following specializations occur ${ }^{1}$ :

Case I: $\quad X=1, Y=Y\left(x^{1}\right), F=F\left(x^{1}\right), h=0$,

Case II: $h=y=0$,

Case III: $F=1, X=X\left(x^{0}\right), Y=Y\left(x^{0}\right), y=0$.

A suitable null tetrad for this line element is

with

$$
\begin{aligned}
k_{a} & =2^{-1 / 2}\left[(1 / F) \delta_{a}^{0}-X \delta_{a}^{1}+(X h-y / F) \delta_{a}^{3}\right], \\
n_{a} & =2^{-1 / 2}\left[(1 / F) \delta_{a}^{0}+X \delta_{a}^{1}-(X h+y / F) \delta_{a}^{3}\right], \\
m_{a} & =2^{-1 / 2} Y\left(\delta_{a}^{2}+\text { it } \delta_{a}^{3}\right),
\end{aligned}
$$

$$
g_{a b}=k_{a} n_{b}+n_{a} k_{b}-m_{a} \bar{m}_{b}-\bar{m}_{a} m_{b} .
$$

A straightforward calculation shows that the Newman-Penrose spin coefficients satisfy

$$
\kappa=\sigma=\tau=0, \quad \nu=\lambda=\pi=0 .
$$

${ }^{1}$ In the statement of this result in [3], the condition $F=F\left(x^{1}\right)$ in Case I was inadvertently omitted (private communication). 
With these simplifications the Ricci identities in Newman-Penrose form immediately imply

$$
\Psi_{0}=\Psi_{1}=0, \quad \Psi_{3}=\Psi_{4}=0 .
$$

Thus $k^{a}$ and $n^{a}$ are repeated principal null congruences and the Weyl tensor is type $[2,2]$. The restrictions $\kappa=\sigma=0, v=\lambda=0$ express the fact that these congruences are geodesic and shearfree (Newman and Penrose [7]) and the proof is complete.

Remark. The solutions for which the principal null congruences are non-expanding are contained in Cases I and III, and are characterized by $\partial Y / \partial x^{1}=0$ in Case I, and $\partial Y / \partial x^{0}=0$ in Case III.

It thus follows from [3] that

Corollary. The only locally rotationally symmetric perfect fluid spacetimes with $C_{a b c d} \neq 0$ whose repeated principal null congruences are nonexpanding are the Gödel universe, the particular solution (6.23), (6.24), with $R=$ constant of Case II a (\$6) and the solution (6.38), (6.39) which is the general solution in Case II b (§6).

\section{§ 8. Conclusion}

The cases I, II a and II b of $\S 5$ and $\S 6$ comprise all algebraically special perfect fluid spacetimes whose repeated principal null congruence is geodesic, shearfree and non-expanding (class A of the introduction).

As an immediate consequence of Eq. (4.22), we can state

Theorem 8.1. The solutions of Case I comprise all algebraically special perfect fluid spacetimes whose repeated principal null direction is parallel to a (null) Killing vector.

From $\S 6$, it is clear that zero pressure is not possible in Cases II a and II b. In Case I, the assumption of zero pressure led uniquely to the Gödel solution and the conformally flat Einstein static solution. Thus we can give a new characterization of the Gödel solution.

Theorem 8.2. The only algebraically special (but not conformally flat) solution of the Einstein field equations for dust, whose repeated principal null congruence is geodesic, shearfree and non-expanding is the Gödel solution.

\section{References}

1. Pirani, F. A. E.: In: Bondi, Pirani, Trautman: Lectures on general relativity. Englewood Cliffs, N. J.: Prentice Hall 1964.

2. Ellis, G. F. R.: Dynamics of pressure-free matter in general relativity. J. Math. Phys. 8, 1171-1194 (1967). 
3. Stewart, J. M., Ellis, G. F. R.: Solutions of Einstein's equations for a fluid which exhibit local rotational symmetry. J. Math. Phys. 9, 1072-1082 (1968).

4. Kantowski, R., Sachs, R. K.: Some spatially homogeneous anisotropic relativistic cosmological models. J. Math. Phys. 7, 443-446 (1966).

5. Wahlquist, H. D.: Interior solutions for a finite rotating body of perfect fluid. Phys. Rev. 172, 1291-1296 (1968).

6. Szekeres, P.: On the propagation of gravitational fields in matter. J. Math. Phys. 7, 751-761 (1966).

7. Newman, E., Penrose, R.: An approach to gravitational radiation by a method of spin coefficients. J. Math. Phys. 3, 566-578 (1962).

8. - Tamburino, L.: Empty space metrics containing hypersurface orthogonal geodesic rays. J. Math. Phys. 3, 902-907 (1962).

9. Talbot, C. J.: Newman-Penrose approach to twisting degenerate metrics. Commun. math. Phys. 13, 45-61 (1969).

J. Wainwright

Department of Applied Mathematics

University of Waterloo

Waterloo, Ontario, Canada 